\title{
Effects of Prophylactic Knee Orthoses Use among Ice Hockey Players in Switzerland
}

\author{
Elisabeth Kupferschmied, Gwendoline Matthey-Doret*, Sébastien Guinault, Daniele Marghitola and Nicolas \\ Mathieu \\ School of Health Sciences, Physiotherapy, HES-SO University of Applied Sciences and Arts Western Switzerland, Switzerland \\ *Corresponding author: Gwendoline Matthey, Physiotherapist, School of Health Sciences, Physiotherapy, HES-SO University of Applied Sciences and \\ Arts Western Switzerland, 3954 Loèche-les-Bains, Switzerland
}

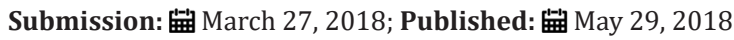

Keywords: Retrospective epidemiological pilot study; Recurrent injury; ACL; MCL; Prophylactic orthosis

\section{Introduction}

Practicing ice hockey can cause a high number of injuries due to frequent physical contact between players, playing surface and material such as puck, boards and sticks. Tegner \& Lorentzon [1] reported that $74 \%$ of the injuries occurred during games and $26 \%$ during practice. The injuries were localized to the knee in $13.2 \%$. The use of prophylactic knee orthoses is very common among worldwide ice hockey players to prevent recurrent lesions of the medial collateral ligament (MCL) and the anterior cruciate ligament (ACL). However, their effects are still widely discussed in the literature owing to the low level of evidence in studies and discrepancy in results.

\section{Purpose of the Study}

The purpose of this study was to assess the feasibility of a given protocol (recruitment, resources, data management, surveys...) for a future national study, and to present the preliminary results of the prophylactic orthotics effects on recurrent injuries ice hockey players in Switzerland.

\section{Methods}

Retrospective epidemiological pilot study, developed according to Thabane's [2] recommendations. Inclusion criteria were all team players of the second and third divisions in Wallis, except for goalkeepers and underage players. Players were asked to report their injuries concerning both knees through a questionnaire. Initial and recurrent injuries were defined as occurring during preseason or regular season games, keeping the player from his activity for at least a day, needing medical consultation and diagnosed with magnetic resonance imaging (MRI) scan. Recurrent injury represented a lesion which appears for the second time on the same knee and same structure (ACL or MCL).
Feasibility was assessed based on previously defined criteria: Participation rate $\geq 75 \%$, ACL-MCL injury rate $>10 \%$ of all knee injuries, Recurrent injury rate $>10 \%$ of specific ACL-MCL injuries, missing data rate $<20 \%$, Charges correlate $+/-5 \%$ pre-established budget and interview duration $<20$ minutes. Exposure times correspond to the number of played matches, with an estimation of 0.3 hours of actual play time per game. Recurrent injuries were divided into three groups: (GA: without orthosis; GB: periodic orthosis; GC: permanent orthosis). Quantitative data, incidence of recurrent injuries and incidence ratio were calculated per 1000 hours of game using the Stata ${ }^{\circledR}$ software program. Data were analyzed using a 95\% confidence interval. Concerning the interpetation of results, if IRR were close to zero, then the risk of recurrent injury with orthosis was lower. Results were statistically significant with $\mathrm{P}$ value $\leq 0.05$.

\section{Result}

Four out of five feasibility criteria were fulfilled. Quantitative data shows that $66.22 \%$ of the target group $(n=80)$ were present and took part in the study. The ACL-MCL injury rate was 48,1\%. Twenty-five MCL and ACL injuries (68\% orthosis wearers) were included, among which $28 \%$ were recurrences. The missing data rate was $5,4 \%$. Recurrent injuries incidence rate was 6,267 per 1000 hours of game. Interview duration took between 3 to 10 minutes. Costs were well within budget. Incidence rate ratio (IRR) of $\mathrm{GA}$ on $\mathrm{GB}=0.153$ (p=0.021); GA on $\mathrm{GC}=2.34 \mathrm{e}-08$ ( $\mathrm{p}=0.00)$.

\section{Discussion}

Relating to feasibility, only the criteria related to participation rate hasn't been completed. This can be explained by three major factors: 
1) Interviews were performed during play-offs period,

2) Many players were transferred to different teams and

3) Many non-professional players were missing. So that the interview lists built through "eliteprospects.com" were temporarily untrue. The duration of interview was overestimated in the protocol.

Concerning knee injuries and braces, this pilot-study shows that a half of players (50\%) had been affected by a knee injury at least once in their career. Center and Wings players are more likely to have incur MCL-ACL injuries. The result of this pilot study indicates that wearing knee orthosis significantly decrease the risk of recurrent injuries. Indeed, no recurrent injury appeared in the study. However, the sample size is too limited to have a wellfounded conclusion.

\section{Conclusion}

A national study is possible subject to minor changes. Recurrent injuries can be prevented in a significant way by wearing an orthosis. However, due to the small sample size, these results must be interpreted with caution.

\section{References}

1. Tegner Y, Lorentzon R (1991) Ice hockey injuries: incidence, nature and causes. Br J Sports Med 25(2): 87-89.

2. Thabane L1, Ma J, Chu R, Cheng J, Ismaila A, et al. (2010) A tutorial on pilot studies: the what, why and how. BMC Med Res Methodol 10: 1. (c) (i) Creative Commons Attribution 4.0

For possible submissions Click Here

\section{Submit Article}

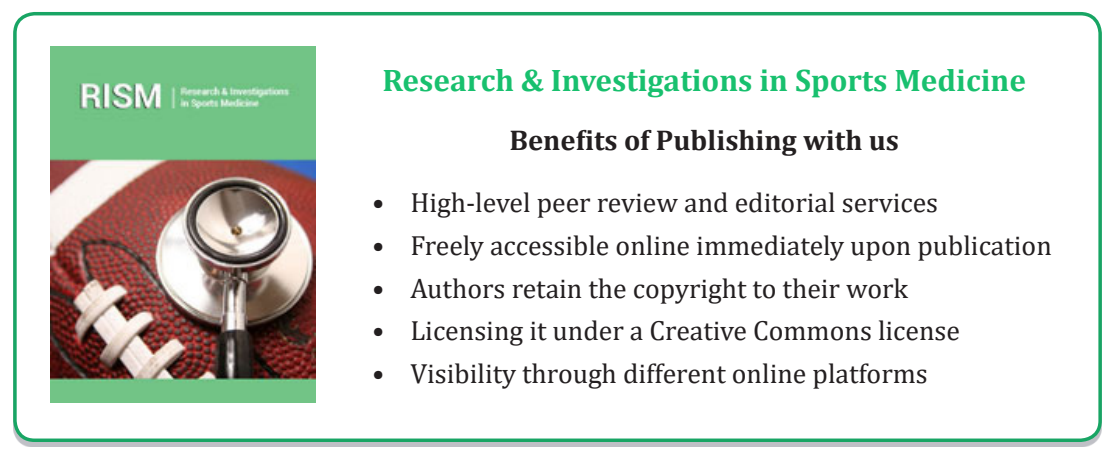

